\title{
AN AUTOZEROING FLOATING-GATE SECOND-ORDER SECTION
}

\author{
Paul Hasler, Theron Stanford, Bradley A. Minch, and Chris Diorio \\ Georgia Institute of Technology \\ Atlanta, GA 30332-0250 \\ phasler@ee.gatech.edu
}

\begin{abstract}
We introduce the Autozeroing Floating-Gate Second-Order Section. We built this second-order filter where the corner frequency and $\mathrm{Q}$ are electronically tunable based on a classic filter topology and principles of OTA amplifiers. We built this second-order filter using three AFGAs - our floating-gate amplifier that sets its operating point by the interaction of hot-electron injection and electron-tunneling.
\end{abstract}

We introduce the Autozeroing Second-Order Section (AutoSOS) in this paper. The second-order section is the primary element to build higher-order filters, both in continuoustime and switch-capacitor circuits. Figure 1 shows the autozeroing second-order section. This circuit is based on the Diff2 second-order section [3], which in turn is based on a canonical form for a second-order section as a high-gain amplifier with feedback. This circuit uses the autozeroing floating-gate amplifier (AFGA) that we invented previously $[1,2]$. The AFGA does not require any clocking to eliminate offsets, as in switched-capacitor circuits [4], but utilizes an inherent combination of electron-tunneling and hot-electron-injection currents to eliminate the DC signal.

This paper illustrates how to build higher-order filters from AFGAs, as well as proving the feasibility of circuits with multiple floating-gate devices. Using AFGAs as the core filter block merges many of the benefits of switchedcapacitor [4], and transconductance-amplifier or OTA [3] filters. The AFGA-based filters can tuned electronically the corner frequencies over many orders of magnitude, as in transconductance-amplifiers. Because many of the AFGA's circuit properties - bandpass gain bandpass gain, linear range, and dynamic range - are set by ratios of capacitors, the AFGA-based filters utilize capacitor matching as is done in switched-capacitor filters. Also, the AFGA filters its offsets, $1 / f$ noise without resorting to the chopper-stabilizing clocking schemes present in switched-capacitor [4] filters.

We present data from circuits fabricated in a $2 \mu m n$-well Orbit CMOS process available through MOSIS. Typical operating values for $V_{t u n}$ were between $33 \mathrm{~V}$ to $42 \mathrm{~V}$; those for $V_{d d}$ were $6 \mathrm{~V}$ to $12 \mathrm{~V}$. We obtain similar data in the $1.2 \mu \mathrm{m} \mathrm{n}$ well Orbit CMOS process, but with typical operating values for $V_{t u n}$ between $26 \mathrm{~V}$ and $31 \mathrm{~V}$. For processes with smaller line widths, the typical operating voltages will decrease due to the thinner gate oxide and higher substrate doping.

\section{AUTOZEROING FLOATING-GATE AMPLIFIER}

Figure 2 shows the AFGA. The AFGA has four oper-

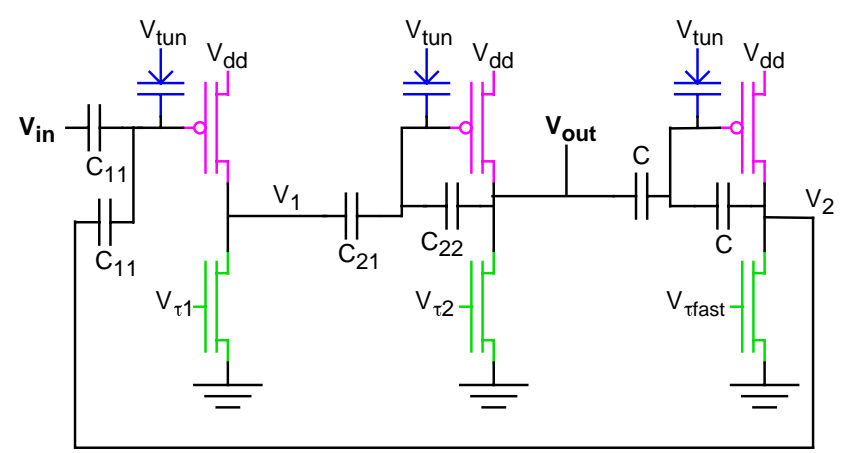

Figure 1: Circuit diagram of the autozeroing second-order section. This circuit, which is built with three autozeroing amplifiers, shows second-order behavior that is electronically controlled.

ating regimes which behave similarly for above and below threshold biases. First, the adaptation region of the AFGA is a high-pass filter at low frequencies, which is set by the tunneling and injection currents. The complementary combination of tunneling and hot-electron injection adjusts the floating-gate charge in such a way that the amplifier's output voltage returns to its steady-state value on a slow time scale. We show elsewhere that the low-frequency AFGA behavior is described by $[1,2]$

$$
C_{2} \frac{d V_{\text {out }}}{d t}=-C_{1} \frac{d V_{\text {in }}}{d t}+I_{\text {tu } 0}\left(\exp \left(-\frac{\Delta V_{\text {out }}}{V_{\text {inj }}}\right)-1\right) .
$$

We derive the frequency response by keeping only the linear terms when we expand the exponentials and taking the Laplace transform:

$$
\frac{V_{\text {out }}(s)}{V_{\text {in }}(s)}=-\frac{C_{1}}{C_{2}} \frac{s \tau_{l}}{1+s \tau_{l}},
$$

where $\tau_{l}$ is $C_{2} V_{i n j} / I_{t u n 0}$. The difference between the tunneling voltage and the pFET's floating gate voltage sets the equilibrium tunneling current, $I_{t u n} 0$, and sets the resulting corner frequency.

Second, the integrating region of the AFGA is a lowpass filter at high frequencies, which is set by the $n$ FET bias current. We show elsewhere that the high-frequency 


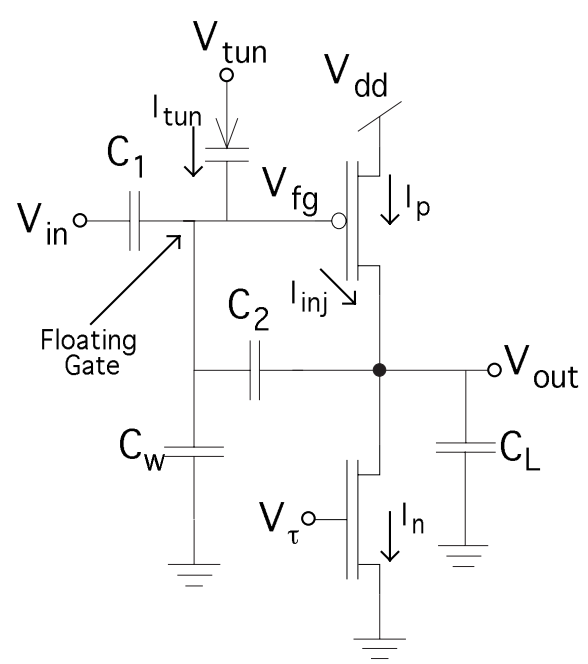

(a)

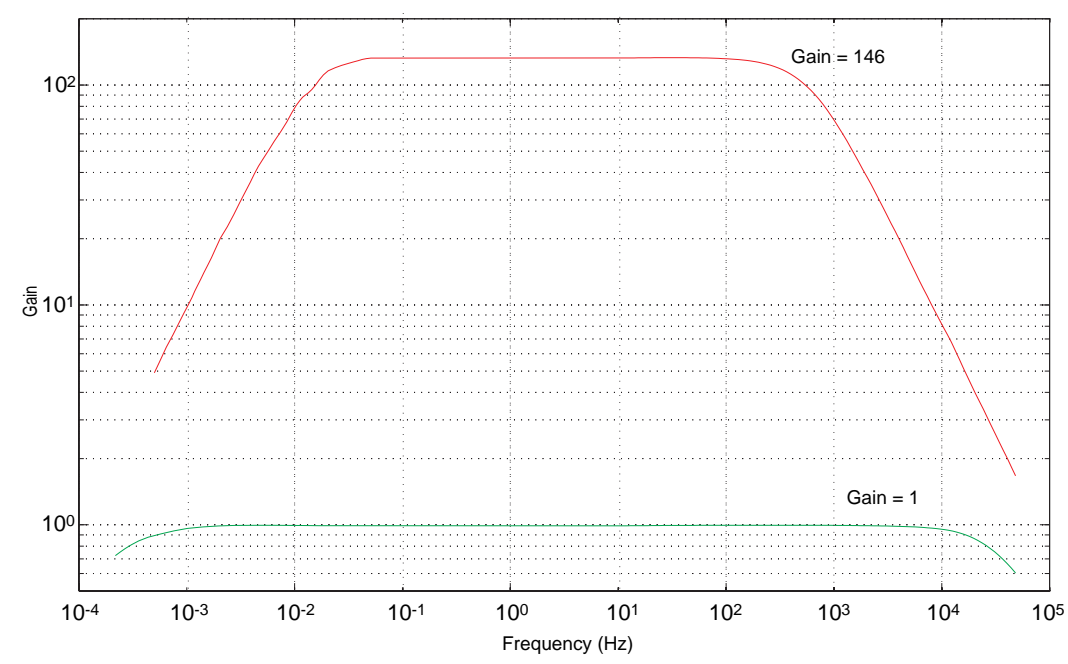

(b)

Figure 2: The autozeroing amplifier that uses the hot-electron injecting $p$ FET. (a) Circuit of The ratio of $C_{2}$ to $C_{1}$ sets the gain of this inverting amplifier. The $n$ FET is a current source, and it sets the current through the $p$ FET. Steady state occurs when the injection current $\left(I_{i n j}\right)$ is equal to the tunneling current $\left(I_{t u n}\right)$. The capacitance from the floating gate to ground, $C_{w}$, represents both the parasitic and the explicitly drawn capacitances. Increasing $C_{w}$ will increase the linear input range of the circuit. The capacitance connected to the output terminal, $C_{L}$, is the load capacitance associated with driving other amplifiers. (b) Frequency response for two AFGAs with different gains. For both the high- and low-gain AFGA, $C_{1}+C_{2}$ is approximately constant. For the high-gain AFGA, $\tau_{l}$ is $20 \mathrm{mHz}$, and $\tau_{h}$ is $600 \mathrm{~Hz}$; for the low-gain $\mathrm{AFGA}, \tau_{l}$ is $300 \mu \mathrm{Hz}$ and $\tau_{h}$ is $40 \mathrm{kHz}$. The ratio of $\tau_{h}$ and $\tau_{l}$ between the two AFGAs are equal to one-half of the ratio of the gains; the ratio is consistent with a constant $C_{1}+C_{2}$.

AFGA behavior is described by [2] which we write as

$$
\left(C_{T} C_{o}-C_{2}^{2}\right) \frac{d V_{f g}}{d t}=C_{1} C_{o} \frac{d V_{i n}}{d t}+C_{2} I_{\tau}\left(e^{-\frac{\kappa \Delta V_{f g}}{U_{T}}}-1\right)
$$

where $C_{T}$ is the total capacitance connected to the floating gate $\left(C_{T}=C_{1}+C_{2}+C_{w}\right)$, and $C_{o}$ is the total capacitance connected to the output node $\left(C_{o}=C_{2}+C_{L}\right)$. Again, we derive the frequency response by keeping only the linear terms when we expand the exponentials and taking the Laplace transform:

$$
\frac{V_{\text {out }}}{V_{\text {in }}}=-\frac{C_{1}}{C_{2}} \frac{1-A_{h} \tau_{h} s}{1+\tau_{h} s}
$$

where we define

$$
\tau_{h}=\frac{\left(C_{T} C_{o}-C_{2}^{2}\right) U_{T}}{\kappa C_{2} I_{\tau}}, \quad A_{h}=\frac{C_{1}}{C_{T}-\frac{C_{2}^{2}}{C_{T}}} .
$$

The gain from input to output due to capacitive feedthrough is $A_{h}$. This transfer function includes the effects of parasitic and load capacitances. The nFET current source sets the bias current and sets the resulting corner frequency.

Third, the amplifier region of the AFGA is between the adaptation and integration regimes. With capacitive feedback, the input signal is amplified by a closed-loop gain approximately equal to $-\frac{C_{1}}{C_{2}}$. Fourth, the capacitive feedthrough region occurs at much higher frequencies than the integrating regime, which can be reduced by increasing either $C_{w}$ or $C_{L}$.

The AFGA is always a first order system even in the presence of parasitic capacitances; therefore, the AFGA is always stable with 90 degrees of phase margin for noninductive loads. Figure $2 \mathrm{~b}$ shows the frequency response from typical high-gain and unity-gain AFGAs. Any amplifier with resistive feedback is at least a second-order system, but amplifiers with capacitive feedback can be a first-order system. For moderate adaptation rates, the low-frequency time constant remains nearly constant, and any shift is primarily due to trapping in the tunneling oxide.

The AFGA's noise performance is similar in thermal and 1/f noise characteristics to a standard MOS amplifier; the tunneling and injection processes do not add appreciable noise to the amplifier. In addition, the adaptation in the AFGA filters the low frequency noise in the AFGA, an effect which can not be obtained in a simple amplifier with a blocking capacitor at the input We can increase the linear range by increasing $C_{w}$, and we can increase the dynamic range by increasing either $C_{w}$ and/or $C_{L}$.

\section{AUTOZEROING SECOND-ORDER SECTION}

Figure 1 shows the autozeroing second-order section. To build a simplified model of the second-order behavior, we assume that $V_{2}=-V_{\text {out }}$; that is, $V_{\tau \text { fast }}$ is set at a bias current much larger than $V_{\tau 1}$ or $V_{\tau 2}$. We ignore (for the moment) the floating-gate currents, ignore the capacitive 


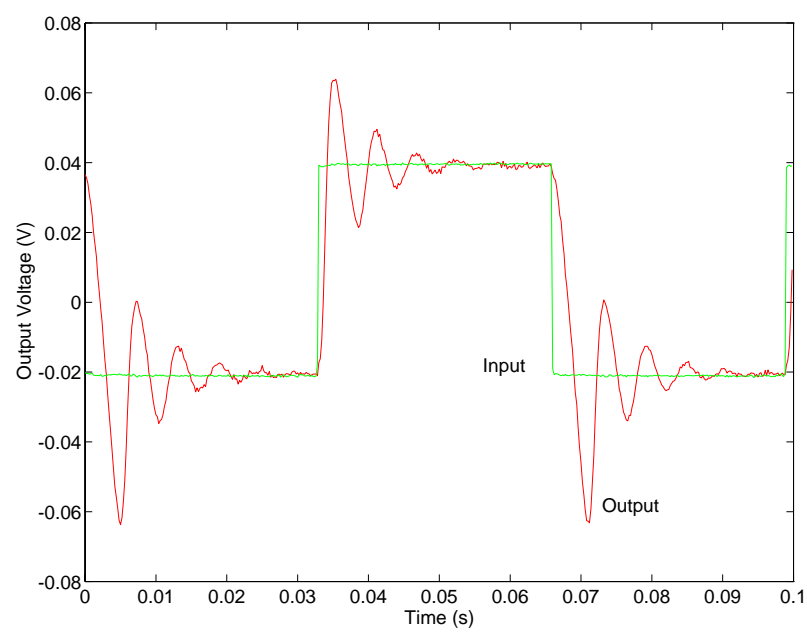

Figure 3: Response of the autozeroing second-order section due to upgoing and downgoing input steps over a short timescale. The ringing of the output voltage is characteristic of a second-order system.

feedthrough effects, and assume the $V_{\tau 1}$ amplifier's gain is very large. From the AFGA analysis in Section 1, the smallsignal model of the two amplifiers is

$$
\begin{gathered}
\frac{C_{T 1} C_{o 1}-C_{21}^{2}}{C_{21}} \frac{U_{T}}{\kappa I_{\tau 1}} \frac{d V_{1}}{d t}=\left(V_{\text {in }}+V_{2}\right), \\
\frac{C_{T 2} C_{o 2}-C_{22}^{2}}{C_{22}} \frac{U_{T}}{\kappa I_{\tau 2}} \frac{d V_{\text {out }}}{d t}=\left(V_{1}+V_{\text {out }}\right),
\end{gathered}
$$

where $C_{21}, C_{22}$ are the feedback capacitors from floating gate to output. By defining

$$
\tau_{1}=\frac{C_{T 1} C_{o 1}-C_{21}^{2}}{C_{21}} \frac{U_{T}}{\kappa I_{\tau 1}}, \text { and } \tau_{2}=\frac{C_{T 2} C_{o 2}-C_{22}^{2}}{C_{22}} \frac{U_{T}}{\kappa I_{\tau 2}},
$$

we solve for the transfer function from $V_{\text {in }}$ to $V_{\text {out }}$ as

$$
\frac{V_{\text {out }}(s)}{V_{\text {in }}(s)}=\frac{1}{1+s \tau_{1}+s^{2} \tau_{1} \tau_{2}} .
$$

From the canonical form for the second-order section, we find that [3] $\tau=\sqrt{\tau_{1} \tau_{2}}$, and $Q=\sqrt{\tau_{1} / \tau_{2}}$. Figure 3 shows the step response of this autozeroing second-order section. The data show the characteristic ringing behavior of a second-order system; changing $V_{\tau 1}$ and $V_{\tau 2}$ changes $\tau$ and $Q$, as predicted from (2).

Figure 4 illustrates how the frequency response changes by electronically changing the $\tau_{1}$ and $\tau_{2}$ values in an AutoSOS; Fig. 4 shows the frequency response for two different values of $\tau_{1}$ and $\tau_{2}$. We tuned these values such that the product of $\tau_{1} \tau_{2}$, and therefore $\tau$, remained constant. In these curves, one sees an initial 20dB/decade increase just before the $\mathrm{Q}$ peak in both responses due to the cutoff frequency of the third amplifier, and this effect increases the amplitude at the $\mathrm{Q}$ peak. This response is typical of the $V_{1}$ node in an ideal second-order section. The capacitive
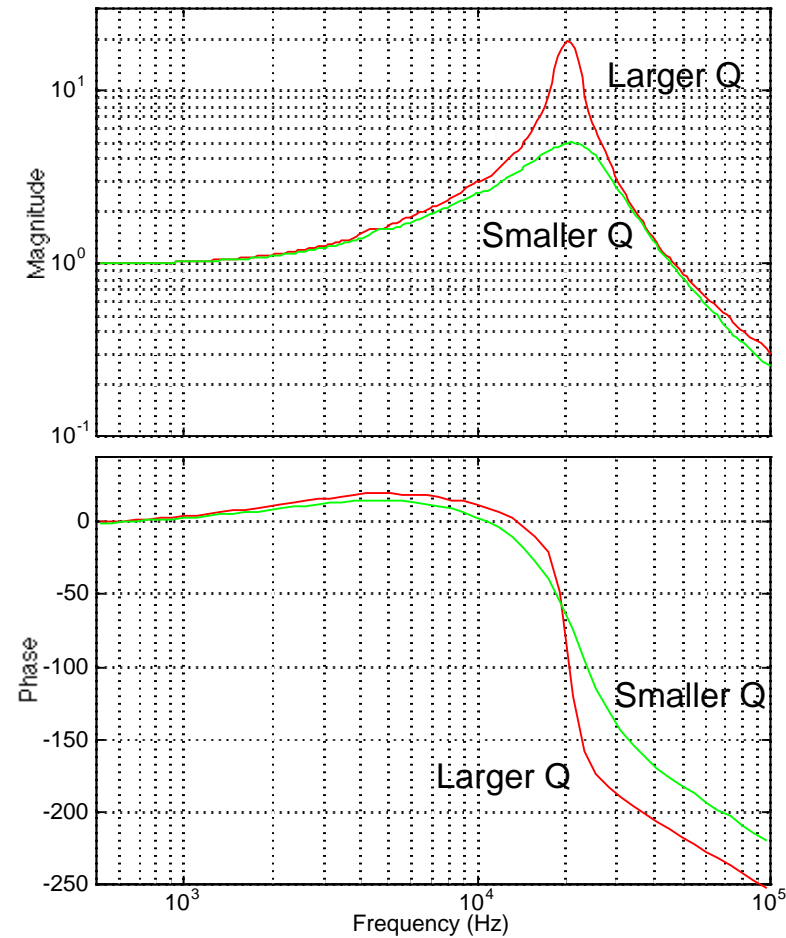

Figure 4: Frequency response of an AutoSOS for two different bias current settings. The Larger $\mathrm{Q}$ case is for $V_{\tau 1}$ $=0.717 \mathrm{~V}$ and $V_{\tau 2}=0.597 \mathrm{~V}$, and the Smaller Q case is for $V_{\tau 1}=0.677 \mathrm{~V}$ and $V_{\tau 2}=0.637 \mathrm{~V}$. In both cases, we changed the bias current from the model of $\tau$ and $Q$ such that the $\tau$ of the circuit would remain constant.

feedthrough in the third amplifier limits the bandwidth of this effect. The high-frequency phase lag greater than 180 degrees occurs because we are using three amplifiers to build a second-order system.

Figure 5 shows the frequency response of the first and second harmonics from identical AutoSOSs except with different drawn sizes of $C_{w}$ (capacitance from floating gate to $\left.V_{d d}\right)$ in all three AFGAs. The second harmonic dominates the distortion of the AFGAs, due to circuit asymmetries, and therefore the distortion of the AutoSOS; the maximum second-harmonic distortion is nearly at half of the section's corner frequency. Increasing $C_{w}$ has two effects:

1. $\tau$ decreases linearly with increasing $C_{w}$, and

2. Second-harmonic distortion decreases linearly with increasing $C_{w}$.

Therefore, as in the AFGAs [2], the AutoSOS's linear range and dynamic range increases linearly and peak distortion decreases linearly with increasing $C_{w}$.

\section{LOW-FREQUENCY TIME-RESPONSE BEHAVIOR}

The AFGA also has a high-pass cutoff at low frequencies; therefore coupling three of these stages with negative feedback may also produce second or third order behavior at low 


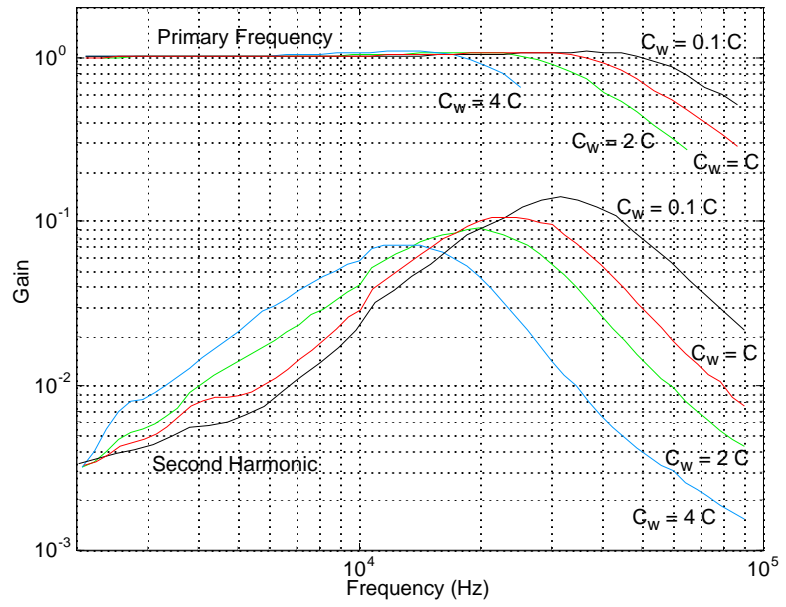

Figure 5: Frequency response of the first and second harmonic components of AutoSOSs for four different values of $C_{w}$ in all three AFGAs. The AutoSOS was biased to have a Q slightly greater than 1 . The input amplitude was much larger than the second AFGA's linear range. We see that increasing $C_{w}$ linearly increases $\tau$, but linearly decreases the peak second-harmonic response.

frequencies. Using small signal models, we can write three equations for the three AFGAs around the low-frequency corners as (assume for clarity $C=C_{11}=C_{21}=C_{22}$ )

$$
\begin{gathered}
\frac{d V_{2}}{d t}=-\frac{d V_{\text {in }}}{d t}-\frac{I_{\text {tun } 01}}{V_{\text {in } j} C} \Delta V_{1} \\
\frac{d V_{1}}{d t}=-\frac{d V_{\text {out }}}{d t}-\frac{I_{\text {tun } 02}}{V_{\text {inj }} C} \Delta V_{\text {out }} \\
\frac{d V_{2}}{d t}=-\frac{d V_{\text {out }}}{d t}-\frac{I_{\text {tun } 03}}{V_{\text {inj }} C} \Delta V_{2}
\end{gathered}
$$

where the equilibrium tunneling currents, $I_{t u n 01}, I_{t u n 02}, I_{t u n 03}$, are set by difference between their respective tunneling and pFET's floating-gate voltages. We have ignored the passband regime for the first amplifier, because it has a small effect on the low-frequency dynamics, as was true for deriving the high-frequency dynamics. Laplace transforming these equations, we solve the coupled equations for the following resulting $V_{2}$ equation:

$$
V_{2}=-\frac{V_{i n}}{1+\frac{I_{t u n 01}}{s C V_{i n j}}+\frac{I_{t u n 01}\left(I_{t u n 02}+I_{t u n 03}\right)}{s^{2} C^{2} V_{i n j}^{2}}+O(3)} .
$$

By comparing this equation to the low-frequency equivalent of a second-order section:

$$
\frac{V_{2}(s)}{V_{i n}(s)}=\frac{1}{1+\frac{1}{s \tau}+\frac{1}{s^{2} \tau^{2}}} .
$$

we get an effective $\tau$ and $Q$ as

$$
\tau=\frac{\sqrt{I_{t u n 01}\left(I_{t u n 02}+I_{t u n 03}\right)}}{C V_{i n j}} \text { and } Q=\sqrt{\frac{I_{t u n 02}+I_{t u n 03}}{I_{t u n 01}}}
$$

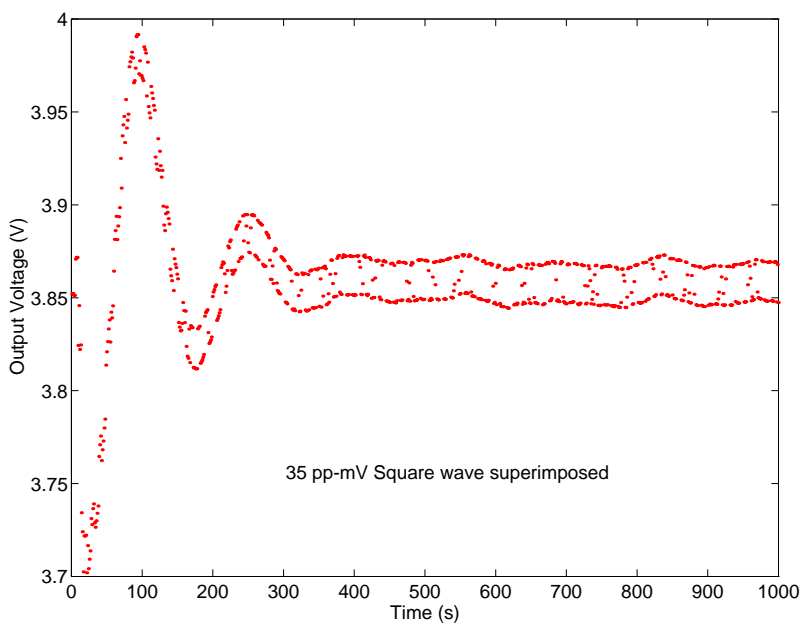

Figure 6: Response of the autozeroing second-order section due to an upgoing input steps over a long timescale. A $1 \mathrm{~Hz}$ square wave was superimposed on the input signal, and is preserved throughout the relaxation. This ringing behavior proves that the circuit exhibits at least secondorder behavior from the AFGA corner frequencies set by the floating-gate currents.

Figure 6 shows the step response at a very slow timescale. The dampled oscillatory behavior is due to the corner frequencies set by the tunneling currents, and is indicative of at least a second-order circuit. The low-frequency filtering in this circuit provides the basis for the design of integrated filters operating at very low frequencies. A complete modeling of either the high or low frequency operation is difficult since both sets of dynamics are really third order.

\section{ACKNOWLEDGMENTS}

This work was supported by the NSF ERC Center for Neuromorphic Systems Engineering.

\section{REFERENCES}

[1] P. Hasler, B.A. Minch, C. Diorio, and C. Mead, "An autozeroing amplifier using $p$ FET hot-electron injection", Proceedings of the International Symposium on Circuits and Systems, Atlanta, vol.3, 1996, pp. 325328. Also at http://www.ee.gatech.edu/users/phasler

[2] P. Hasler, B.A. Minch, C. Diorio, and C. Mead, "An autozeroing floating-gate amplifier", Accepted for publication in Circuits and Systems II: Digital and Analog Signal Processing

[3] C. Mead, Analog VLSI and Neural Systems, Addison - Wesley, Reading, MA, 1989.

[4] R. Gregorian and G.C. Temes, Analog MOS Integrated Circuits for Signal Processing, Wiley-Interscience, New York, NY, 1986. 